\title{
A Multiscale Neural Network Method for Image Restoration
}

A.P.A. de CASTRO, I.N. DRUMMOND, J.D.S. da SILVA, ${ }^{1}$ Laboratório Associado de Matemática e Computação Aplicada, LAC, INPE, 12227-010 São José dos Campos, SP, Brasil.

\begin{abstract}
This paper describes a novel neural network based multiscale image restoration approach. The method uses a Multilayer Perceptron (MLP) trained with synthetic gray level images of artificially degraded co-centered circles. The main difference of the present approach to existing ones relies on the fact that the space relations are used and they are taken from different scales, which makes it possible for the neural network to establish space relations among the considered pixels in the image. This approach attempts at coming up with a simple method that leads to an optimum solution to the problem without the need to establish a priori knowledge of existing noise in the images. The multiscale data is acquired by considering different window sizes around a pixel. The performance of the proposed approach is close to existing restoration techniques but it was observed that the resulting images showed a slight increase in contrast and brightness. The proposed technique is also used as a preprocessing phase in a real-life classification problem of medical Magnetic Resonance Images (MRI) by using a fuzzy classification technique.
\end{abstract}

Key-words. Image Restoration, Artificial Neural Networks, Image Classification.

\section{Introduction}

Image restoration is an inverse problem stated as the recovery of an image from its probably degraded version. Existing methods usually require a priori knowledge of the degradation process to design the solution that may compensate for the degradation problems caused by motion blur, atmospheric turbulence, and optical diffraction [13].

The degradations may result from noise in the sensor, loss of focus, objectcamera relative motion, and random atmospheric turbulence [12]. Such noise sources in images are characterized by Gaussian-like distributions [11].

In general restoration techniques are oriented towards the recovery of the real image by applying a restoration process to its degraded version $[9,1]$. Some common methods for image restoration include the inverse filter, the Wiener filter, the moving-average filter, the parametric Wiener filter, the mean-squared-error filter,

\footnotetext{
${ }^{1}$ (apaula, isabela, demisio)@lac.inpe.br
} 
the band-pass filter and the singular value decomposition technique [9], as well as, the regularization filter [1].

Recently, some of the methods for image restoration have been modified in an attempt to improve their solutions and reduce the computational complexity [6]. Due to the wide use as tools for information processing, Artificial Neural Network (ANN) models have also bee used to design new solutions to the image restoration problem. They present some features that may lead to better results in the image restoration process [14]. Such features are related to its plasticity and their parallel computing power that have made them appropriate for applications in pattern recognition, signal processing, image processing, computer vision, and several other application areas. There exists a number of different neural network architectures well described in the computer science literature.

In this paper we present a novel neural network based multiscale restoration approach [4, 5]. The method uses a Multilayer Perceptron (MLP) algorithm [10], trained with synthetic a 8-bit gray level image of artificially degraded co-centered circles, with $256 \times 256$ pixels.

In order to design the training set for the neural network, the artificially degraded image is submitted to a clustering performed by a Kohonen neural network, using a threshold level of similarity for existing neurons (cluster centers). The algorithm adds a new neuron and assigns the corresponding input vector as its corresponding weights when existing neurons are not able to overcome the threshold level in the competition phase of the Kohonen neural network. This process leads to reduced training data sets, that vary according to the threshold level settings.

The learning phase of the Multilayer Perceptron attempts to make it capture inherent space relations of degraded pixels and correspond them to the non-degraded pixels. In the conducted experiments, the degradation effects are simulated by applying the degradation model in [9]. The image is first convoluted with a lowpass Gaussian filtering operation and then noise is added to it at $1 \%$ rate occurrence. The degraded image data is provided as input to the MLP and the non-degraded image as the corresponding output in the supervised learning process.

The main difference of the present approach to existing ones relies on the use of space relations taken from the vicinity of the considered pixel in different scales, which makes it possible for the neural network to establish space and contextual relations among the considered pixels in the image. This approach attempts to come up with a simple method that leads to an optimum solution to the problem without the need to provide a priori knowledge of existing noise in the images.

In the conducted experiments, the trained neural network is submitted to indoor, outdoor, and satellite degraded images to verify the generalization performance on different image types. The results are compared to existing restoration approaches (focusing the Wiener filter) by varying the similarity parameter in the Kohonen clusterization algorithm used to reduce the input vector.

The paper is organized as follows. Section 2 describes the proposed restoration

method. Section 3 presents some experimental results, with a quantitative analysis. Section 4 presents an application in which the proposed technique is used as a preprocessing phase of real-life classification problem of MRI. Finally, section 5 brings the conclusion for the work. 


\section{The Proposed Approach}

In this paper we present a neural network based image restoration technique using local spatial information acquired in a multiscale approach. The design of the method involves three phases: a) image information extraction; b) data clusterization to reduce the amount of data to form the training set; and c) the MLP training to capture a general inverse reconstruction model.

\subsection{Training data}

The proposed approach assumes the effects of the degradation sources mentioned before are universal in nature, that is, in general images are subject to the same degradation sources, and that they may be simulated on synthetic images. Thus, an 8-bit gray level image of co-centered circles was created and submitted to an artificial degradation process as previously stated. Figure 1.a shows the degraded image on the left and the corresponding non-degraded version on the right.

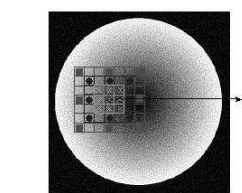

a.

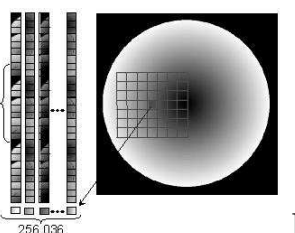

b.

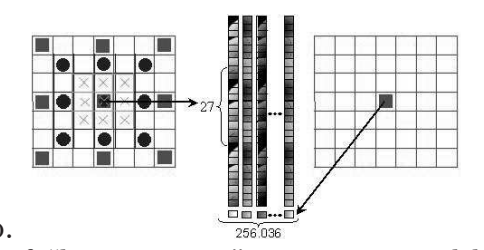

Figure 1: Multiscale approach: a) overall view of "linearization" in process and b) illustration of the $7 \times 7$ window neighborhood subsample method.

The training data set was created by sequentially extracting $3 \times 3,5 \times 5,7 \times 7$ windows from the degraded image and the pixel of the corresponding window center position in the original nondegraded image.

The $5 \times 5$ and $7 \times 7$ were subsampled to form two $3 \times 3$ windows to simulate the multiscale approach. Then, three $3 \times 3$ windows data were then gather to form the input vector for training purposes. The pixel in the center of the corresponding $7 \times 7$ window in the non-degraded image was chosen to be the desired output in the training process. Both the input vector and desired output pixel formed a 28member vector which was sequentially concatenated to other vectors of the same nature to form the training set (see Figure 1). This process resulted in a very large training data set that may imply in a long time to train the MLP in the modeling process. Thus, the previously cited clusterization process using a Kohonen neural network was applied in a data mining like approach to reduce the amount of data for training purposes.

\subsection{Data mining}

The cited data mining process consisted of designing a clustering algorithm that was implemented as a Kohonen neural network. A similarity Gaussian metric was implemented. The idea was to establish a threshold limit to the competition phase 
of the learning processo of the Kohonen neural neural. The winning neuron had to overcome the threshold (robustness) to have its weight updated.

If the winner neuron did not overcome this threshold, a new neuron had to be inserted in the one-dimensional lattice to represent the input vector whose elements were assigned to the corresponding elements of the weight vector (See Figure 2).

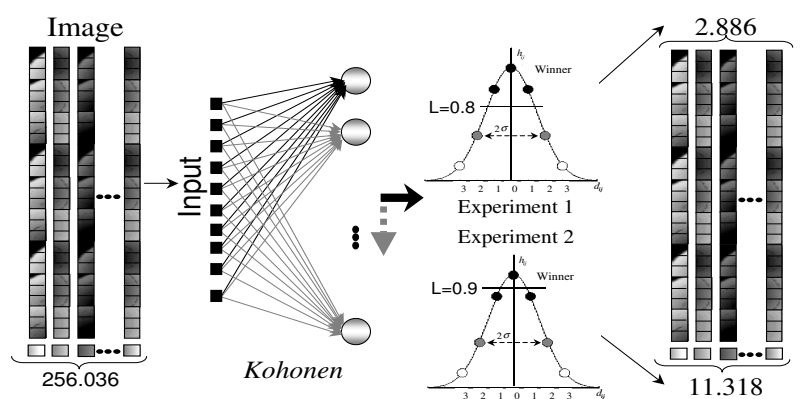

Figure 2: Clustering process

In this work two similarity thresholds were used: a 0.9 and 0.8 of a Gaussian transformation of the distance of the weight vector of each neuron to the input vector. Table 1 shows the size data vectors.

Table 1: Training set reduction

\begin{tabular}{lcccc}
\hline Image & Size Image & Size & Similarity $80 \%$ & Similarity $90 \%$ \\
\hline circle & $506 \times 506$ & 256.036 & 11.318 & 2.886 \\
\hline
\end{tabular}

\section{3. $\quad$ Training process}

In the training process the degraded image data was provided as inputs to the mutilayer perceptron and the non-degraded image data as the corresponding desired output for the supervised learning process. The MLP was designed with only 1 hidden layer with 14 neurons, each neuron with a logistic sigmoidal activation function.

The main objective to the present approach is to derive a simple image restoration method based on an inverse degradation model that may lead to an optimum solution to the problem.

\section{Experiments}

In this section, we present the results of some experiments conducted with the proposed technique to restore degraded images. Different neural network architectures were trained and then submitted to degraded versions of different images, such as: the Lenna image and an Ikonos satellite image, submitted to the same noise sources used in the training image; and MR brain images from a real data base containing multiple sclerosis lesions.

The use of different image data aimed to verify the adequacy and robustness of the neural network approach to the image restoration problem as stated in [9]. 
A comparison of the proposed method and the Wiener filter was performed through a quantitative analysis by calculating image statistics representing the mean brightness and contrast: the image mean $\left(\mu_{f}\right)$, and the variance $\left(v_{f}\right)$. In addition, the standard deviation $(\sigma)$, the Mean Square Error (MSE), and the Signal-to-NoiseRatio (SNR) are calculated [12]. The intensity mean, variance and standard deviation are properties frequently used in image processing due to their relevance to characterize the appearance of an image [9]. The mean is a measure of the average brightness and the variance is a measure of contrast. The standard deviation is the square root of the variance, which may be interpreted as a measure of image homogeneity.

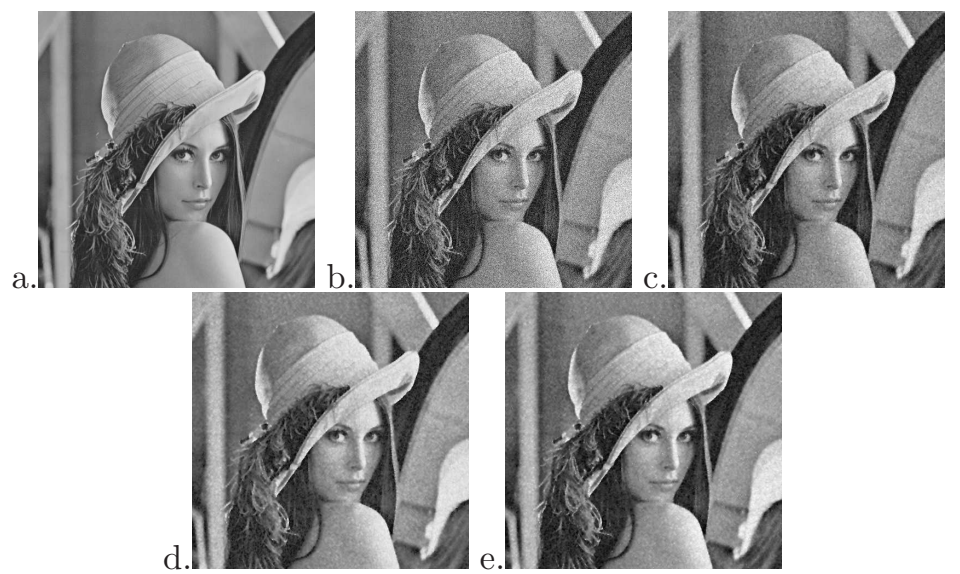

Figure 3: Generalization tests with Lenna image: a) original image; b) Gaussian degraded with $1 \%$ noise; c) restored image by a $3 \times 3$ Wiener filter; d) ANN result - multiscale approach, 80\% similarity; and e) ANN result - multiscale approach, 90\% similarity.

Figure 3 shows the Lenna image: a) without noise; b) artificially degraded using $1 \%$ of noise and after the restoration by c) the Wiener method; and by the neural network approach using the d) $80 \%$ and e) $90 \%$ vector similarity thresholds in the clusterization phase. The quantitative analysis is presented in Tables 2 and 3.

Table 2 brings the results for $\mu_{f}, v_{f}$ and $\sigma$ for the original, degraded and restored images. We can observe an increase in brightness and contrast for the images restored by the proposed approach, through the values obtained for $\mu_{f}$ and $v_{f}$. A non significative difference in the $\sigma$ values shows preservation of image homogeneity.

Table 2: Statistics of the images.

\begin{tabular}{clllll}
\hline Lenna & \multicolumn{1}{c}{ OI } & $1 \%$ noise & \multicolumn{1}{c}{ WF } & ANN 80\% & ANN 90\% \\
\hline$\mu_{f}$ & 24.152 & 124.136 & 123.685 & 120.896 & 125.219 \\
$v_{f}$ & 2295.997 & 2912.072 & 2318.443 & 2208.260 & 2655.231 \\
$\sigma$ & 47.916 & 53.963 & 48.150 & 46.992 & 51.528 \\
\hline
\end{tabular}

The obtained values for MSE and SNR between the original image and the 
degraded version, and between the original and restored images are presented in Table 3. A better performance of the ANN restoration can be verified by comparing its smaller MSE and its larger SNR, with the corresponding values for Wiener restorarion. A smaller MSE means that the restored image is closer to the original version.

Table 3: Filtering errors.

\begin{tabular}{lcccc}
\hline \multicolumn{1}{c}{ Lenna } & MSE & $\%$ & SNR $(\mathrm{db})$ & $\%$ \\
\hline D x OI & 25.278 & - & 5.554 & - \\
WF x OI & 11.141 & - & 12.670 & - \\
ANN 80\% x OI & 10.290 & 7.63 & 13.360 & 5.44 \\
ANN 90\% x OI & 10.708 & 3.88 & 13.015 & 2.72 \\
\hline
\end{tabular}

Figure 4 presents the Ikonos Satellite image: a) without noise, b) artificially degraded using $1 \%$ of noise, and after restoration by c) the Wiener method; and by the ANN approach using a d) $80 \%$ and a e) $90 \%$ vector similarity thresholds in the clusterization phase. The quantitative analysis is presented in Tables 4 and 5 .

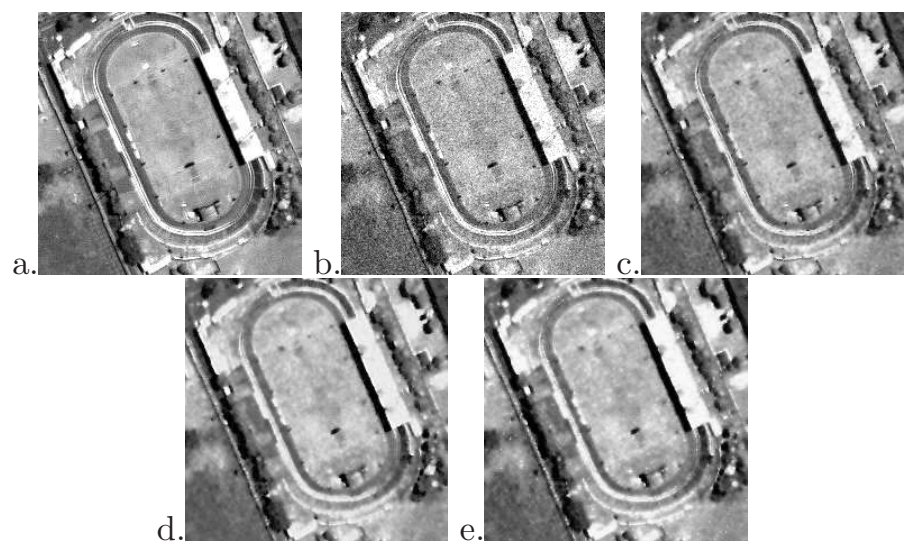

Figure 4: Generalization tests with the IKONOS satellite image: a) original image; b) Gaussian degraded with $1 \%$ noise; c) restored image by a $3 \times 3$ Wiener filter; d) ANN result - multiscale approach, $80 \%$ similarity; and e) ANN result - multiscale approach, $90 \%$ similarity.

In this case, the statistics measurements, presented in Tables 4 and 5, show that the ANN approach led to a good restoration but with a slightly minor performance when compared to the Wiener filter restoration.

Experiments have also been conducted on a real MRI database composed of volumes of $512 \times 512 \times 20$, obtained from a 1-Tesla magnetic field system under the supervision of a radiologist who provided an interpretation of the multiple sclerosis artifacts present in the images. In order to apply the restoration process, the axial T2 and Flair (F) sequences were selected. Figure $5 \mathrm{a}$ ) - b) presents the real MRI T2 (slice 14) and F (slice 15) images without noise, respectively; and after the 
Table 4: Statistics of the images.

\begin{tabular}{clllll}
\hline Ikonos & \multicolumn{1}{c}{ OI } & $1 \%$ noise & \multicolumn{1}{c}{ WF } & ANN 80\% & ANN 90\% \\
\hline$\mu_{f}$ & 144.188 & 143.841 & 143.362 & 146.140 & 147.096 \\
$v_{f}$ & 3515.122 & 3853.715 & 2963.249 & 3011.440 & 3351.115 \\
$\sigma$ & 59.288 & 62.078 & 54.435 & 54.876 & 57.888 \\
\hline
\end{tabular}

Table 5: Filtering errors.

\begin{tabular}{lcccc}
\hline \multicolumn{1}{c}{ Ikonos } & MSE & $\%$ & SNR $(\mathrm{db})$ & $\%$ \\
\hline D x OI & 24.560 & - & 7.654 & - \\
WF x OI & 16.855 & - & 10.924 & - \\
ANN 80\% x OI & 19.620 & -16.40 & 9.605 & 12.07 \\
ANN 90\% x OI & 19.631 & -16.46 & 9.600 & 12.12 \\
\hline
\end{tabular}

restoration by c)- d) the Wiener method; and by the ANN approach using e) - f) $80 \%$ and g) - h) $90 \%$ vector similarity thresholds.

a.
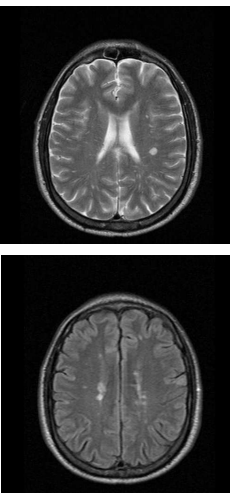

b.
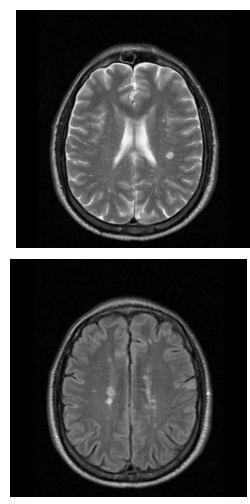
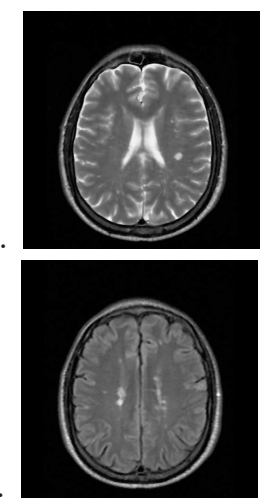

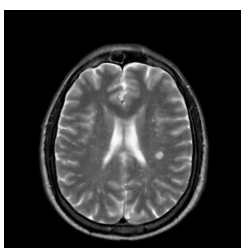

h.

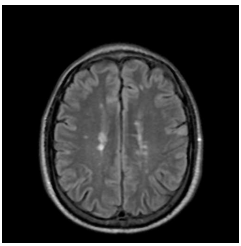

Figure 5: Original MRI (OI), restored by wiener filter (WF) and by multiscale approach (ANN) slices: a) T2_S14 OI; b) T2_S14 WF; c) T2_S14 ANN 80\%; d) T2_S14 ANN 90\%; e) F_S15 OI; f) F_S15 WF; g)F_S15 ANN 80\%; h) F_S15 ANN $90 \%$.

It is to be observed that the original images are not subjected to an artificial degradation procedure. They have been submitted to the restoration approaches as they were acquired. In order to calculate the SNR for the original MR images, we have extracted some regions of interest (ROI) to estimate the noise. We have taken the mean of the variances, considering that this local noise estimation may be observed in the whole image. Thus, there was not a ground truth image available to compare the statistics obtained $\mu_{f}, v_{f}$, and $\sigma$. However, as expected, changes might be observed. To evaluate the performance of the restoration method on such images a fuzzy classification method was proposed in which the feature space is 
checked for improved class sample compactness.

\subsection{Validation of the proposed technique}

As a form of validation of the technique of the proposed restoration approach, a fuzzy classification method was applied to noisy and restored images. The aim of this phase was to verify the benefits of the transformation in the image feature space due to the restoration process applied. It is important to notice that the proposed approach does not consider any prior knowledge of existing degradation problems in the images. The performance of the classifier is analysed by comparison of its application to the noisy images and to their restored versions, using the kappa index [3] to assess the quality of the classifications. We used a prototype based fuzzy classifier [7] that works by first establishing cluster centers for the samples of each class. This is performed by the original fuzzy c-means (FCM) algorithm [2] and a clusterization index [8] is used to calculate the optimal number of centers for each class. The resulting centers are then transformed into fuzzy prototypes by the application of a similarity relation to each one.

We have used two slices, 14 for training and 15 for testing, in sequences T2 and F (see Figures 5a and 5e). Five classes were considered: background, cerebral spinal fluid (CSF), gray matter, white matter, and the Multiple Sclerosis (MS) lesion. The sample extraction was performed under the supervision of a radiologist who established the ground truth for the classification and analysis purposes.

Interestingly, even though the effects of the restoration process are not very evident under visual analysis, as can be seen in Figures 5 b) - d) and f) - h), the classification results were improved in some cases. The MS lesions were diagnosed in a clinical exam using the non-restored images: there are two of them in the right side of the brain in slice 14 and another two in the left side in slice 15.

Table 6 presents the kappa index obtained in the classification of the original image (first column) and in the classification of the restored ones by the Wiener filter and by the multiscale ANN based approach. It is to be noticed that the ANN based approach led to a better classification performance. The explanation to this fact may be associated to low-pass filtering property of the ANN based approach. In the laring phase the input data consists of data from 3 different $3 \times 3$ windows that are linearly combined to lead to the neuron induced local field or activaton potential [10]. The feature spaces, formed by the spectral response of the pixels in $\mathrm{T} 2$ and $\mathrm{F}$ sequences are depicted in Figure 6.

Table 6: Filtering errors.

\begin{tabular}{cccc}
\hline degraded & Wiener & ANN 80\% & ANN 90\% \\
\hline 95.1389 & 95.0000 & 97.9167 & 97.0139 \\
\hline
\end{tabular}




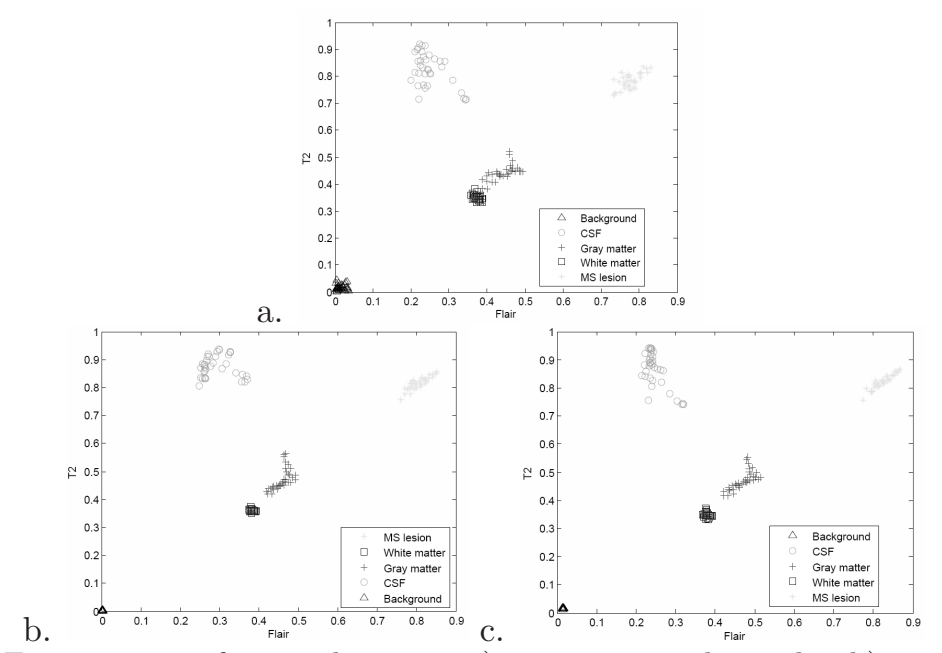

Figure 6: Feature spaces from real images: a) testing original samples; b) testing restored samples using $80 \%$ similarity and c) testing restored samples using $90 \%$ similarity.

\section{Conclusion}

A neural network multiscale image restoration method was proposed for restoring degraded images based on an universal training data strategy. Experimental results show the adequacy of the proposed approach to the problem. Quantitative analysis show the proposed method presented similar results to the ones obtained by using the Wiener filter, reported in the literature as the most used method for image restoration.

However, in most of the conducted experiments the neural network restored images presented a slight enhancement in both brightness and contrast, as observed by the increase in the mean and variance values of the images, while a reduction of the degradation was performed as it may be observed by the signal to noise ratio measurements.

In addition, the performance of the restoration methods discussed and implemented in the paper were also compared by applying such methods to restore a real MR brain image prior to a supervised image fuzzy classification task. Very good results were obtained in the classification for the proposed restoration approach using $80 \%$ and $90 \%$ vector similarity thresholds. The multiscale approach performed better than the classical Wiener filter approach, with the most satisfactory kappa index reached for the image restored by the multiscale ANN based method with $80 \%$ vector similarity threshold. Despite the results obtained, in all of the experiments presented in this paper, the feature space consisted only of the pixels spectral response in different sequences of MRI. The noise reduction produced by the restoration methods, however, may not always guarantee that the accuracy of the classifier will be better. In fact, the image restoration process leads to the classification of different data. However, the compactness effect observed in the feature spaces may be taken as an advantage for the classifier addressed in this paper, 
since it needs less prototypes for each class, thus improving the performance of the classification in terms of computational time.

An advantage of the proposed method is related to the fact a neural network approach may be less computationally expensive than the Wiener filter when dealing with very large image datasets, in addition to the easiness of implementation of the ANN models that may also be implemented directly in an imaging acquisition hardware.

\section{References}

[1] M. Bertero, P. Boccacci, "Introduction to Inverse Problems in Imaging", Philadelphia, Bristol, 1998.

[2] J. Bezdek, R. Ehrlich, W. Full, FCM: The fuzzy c-means algorithm. Computers \& Geosciences, 10, No. 2-3, (1984), 491-263.

[3] Y.M. Bishop, S.E. Feinberg, P.W. Holland, "Discrete Multivariate Analysis: Theory and Practice", Cambridge: MIT Press, 1975.

[4] A.P.A. Castro, J.D.S. Silva, Neural Network-Based Multiscale Image Restoration Approach. In: Proceeding on Electronic Imaging, Vol. 6497, San Jose, pp. 3854-3859, 2007.

[5] A.P.A. Castro, J.D.S. Silva, Neural Network-Based Multiscale Image Restoration Approach. In: Proceedings of IPDO, Miami, 2007.

[6] J. Chen, J. Benesty, Y. Huang, S. Doclo, New insights into the noise reduction wiener filter, IEEE Trans. on Audio, Speech and Language Processing, 14, No. 4 (2006), 1218-1234.

[7] I. Drummond, S. Sandri, A clustering-based possibilistic method for image classification, Lecture Notes in Computer Science, 3171 (2004), 454-463.

[8] I. Drummond, S. Sandri, A clustering-based fuzzy classifier, Frontiers in Artificial Intelligence and Applications, 131, No. 1 (2005), 247-254.

[9] R.C. Gonzalez, R.C. Woods, "Digital Image Processing", New York, Addison Wesley, 1992.

[10] S. Haykin, "Redes Neurais: Princípios e Prática", P. Alegre, Bookman, 2001.

[11] K.V.D. Heijden, "Image Based Measurement Systems", New York, Wiley, 1994.

[12] A.K. Jain, "Fundamentals of Digital Image Processing", New Jersey, Prentice Hall, Inc, 1989.

[13] A.D. Kulkarni, "Computer Vision and Fuzzy-Neural Systems", New Jersey, Prentice Hall, 2001.

[14] Y.D. Wu, Q.Z. Zhu, S.X. Sun, H.Y. Zhang, Image restoration using variational PDE-based neural network, Neurocomputing, 69, No. 16-18, (2006), 2364-2368. 